\title{
Real-World Clinical and Economic Outcomes Associated with Palbociclib for HR-Positive/HER2 Negative Metastatic Breast Cancer: A Commentary
}

\author{
Aram Babcock, PharmD, MBA; Askal Ayalew Ali, PhD; Rajesh Balkrishnan, PhD; \\ Alberto Montero, MD, MBA; and Vakaramoko Diaby, PhD
}

\begin{abstract}
SUMMARY
Despite the achieved advancement in pharmacological cancer treatments, the majority of postmenopausal women with hormone receptor-positive metastatic breast cancer $(\mathrm{mBC})$ will experience disease progression. Research into alternative therapies with improved efficacy and reduced side effects has led to the development of a new class of oral anticancer medications, the cyclin-dependent kinase (CDK) 4/6 inhibitors, which include palbociclib, ribociclib, and abemaciclib. Nonetheless, there is growing evidence that the effectiveness of oral anticancer medications is suboptimal, being influenced by low adherence, sociodemographic factors, and adverse effect profiles. In addition, there is a disconnect between the high price tags of CDK $4 / 6$ inhibitors and their observed effectiveness, raising questions about their value. Currently, the existing knowledge base on the effectiveness and cost-effectiveness of newer oral anticancer medications in understudied populations with possible health disparities is scant. This commentary discusses what is known about palbociclib's clinical effectiveness, safety, and adherence and suggests the need for further studies of real-world effectiveness and cost-effectiveness to help establish the value of newer oncologic drugs, such as palbociclib.
\end{abstract}

J Manag Care Spec Pharm. 2020;26(7):826-31

Copyright $\odot 2020$, Academy of Managed Care Pharmacy. All rights reserved.

$\mathrm{B}$ reast cancer is the second leading cause of cancer-related death among American women. ${ }^{1}$ Breast cancer is a heterogeneous disease composed of distinct molecular subtypes that include luminal A and B, human epidermal growth factor receptor 2 (HER2) type, and triple-negative/basal-like. ${ }^{2}$ As of 2020, the average risk for a woman to develop breast cancer in her life is $13 \%$ in the United States, with an estimated 276,480 new cases of invasive breast cancer and 48,530 new cases of noninvasive breast cancer expected to be diagnosed in 2020. ${ }^{1}$ Breast cancer accounts for approximately $25 \%$ of all cancer cases and $15 \%$ of all deaths among women and constitutes one of the most expensive malignancies to treat. ${ }^{1,3}$ As such, breast cancer puts a heavy burden on patients and their families, as well as health care systems across the world. ${ }^{4}$

Strategies to fight breast cancer are geared toward prevention, early detection, and treatment. ${ }^{5}$ Multiple subtypes and stages of breast cancer have been defined, which has led to the development of multiple, targeted strategies to treat the disease. ${ }^{2}$ Hormone receptor-positive (HR+) and HER2 negative (HER2-) metastatic breast cancer $(\mathrm{mBC})$, together, is the most common subtype, with a highly variable prognosis that depends on the responsiveness to endocrine agents. ${ }^{6}$ Resistance to endocrine therapy (ET), such as aromatase inhibitors (AIs) or fulvestrant, may be the reason for the disease progression experienced by the majority of postmenopausal women with HR+ mBC. Research into alternative therapies with improved efficacy and reduced side effects has led to the development of a new class of oral anticancer medications, the cyclin-dependent kinase (CDK) 4/6 inhibitors, which include palbociclib, ribociclib, and abemaciclib. Nonetheless, there is growing evidence that the effectiveness of oral anticancer medications is suboptimal, being influenced by low adherence and adverse effect profiles. ${ }^{7}$ In addition, there is a disconnect between the high price tags of CDK 4/6 inhibitors and their observed effectiveness, raising questions about their value. ${ }^{8,9}$ Currently, the existing knowledge base regarding the effectiveness and cost-effectiveness of newer oral anticancer medications is scant.

In this U.S. market-focused opinion, we present what is known about palbociclib's clinical effectiveness, safety, adherence, and cost-effectiveness, as well as highlight the need for further studies into real-world effectiveness and cost-effectiveness with adherence in mind.

\section{Adherence and Patient Perspective}

A new weapon in the armamentarium of oral anticancer medications, the CDK 4/6 inhibitors (palbociclib, ribociclib, and abemaciclib) were approved in combination with endocrine therapy (aromatase inhibitors or fulvestrant) as first-line agents to prolong progression-free survival in this subtype. ${ }^{6,10}$ However, it has been shown that a significant number of patients struggle to adhere to oral anticancer medications (OAMs) as prescribed. ${ }^{11}$ As noted in the systematic review by Greer et al. (2016), ${ }_{11}^{11}$ nonadherence to OAMs can lead to lower survival rates, ${ }^{12-14}$ increased risk of recurrence, ${ }^{15,16}$ and higher health care costs. ${ }^{17}$ Also, studies looking at interventions to improve adherence have not been successful because of methodological concerns, resulting in a lack of evidence to guide practice. ${ }^{11}$ For improved patient outcomes, it is essential to properly manage OAM regimens, since most of them have narrow therapeutic ranges and safety margins, making managing medication regimens essential for improved patient outcomes. ${ }^{18}$ Patient perceptions of medications influence 


\section{Real-World Clinical and Economic Outcomes Associated with Palbociclib for HR-Positive/HER2 Negative Metastatic Breast Cancer: A Commentary}

\section{TABLE 1 Description of U.S. Studies that Focus on Real-World Effectiveness and Safety}

\section{Reference}

Varella et al. ${ }^{2}$

\section{Description}

In the 411 women included, the estimated overall median PFS for palbociclib plus letrozole was 8.9 months (6.16, 11.0), and the estimated median PFS was 15.1 months (12.3, not reached) for those who received palbociclib/letrozole as first-line therapy; 10.5 months (7.05, not reached) as second-line therapy; and 4.2 months $(3.7,5.6)$ for third-line and beyond. As for palbociclib/fulvestrant, the estimated overall PFS was 10.3 months $(8.16,12.3)$, with an estimated median PFS of 11.6 ( 8.2 , not reached), 12.3 (8.66, not reached), and 6.4 months $(4.23,11)$, respectively, as first-, second-, or third-line and beyond therapy. The most common AEs were hematologic, with $58 \%$ of patients experiencing grade $3-4$ neutropenia, but only $8 \%$ of the patients permanently discontinued palbociclib due to AEs.

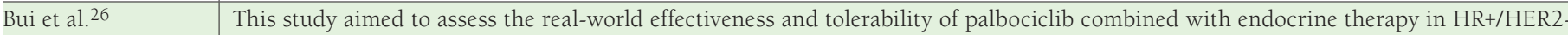
patients with $\mathrm{mBC}$ that progressed on previous endocrine therapy and to compare their results with the outcomes of the PALOMA-3 trial. The median PFS in real-world clinical practice was 10.0 months compared with 9.5 months in PALOMA-3. Most AEs were managed with palbociclib dose modifications, and only 2 patients discontinued treatment because of AEs. Neutropenia was the most frequent grade 3-4 AE, but no febrile neutropenia occurred. Overall, the effectiveness and tolerability of palbociclib in real-world clinical practice were similar to the results from PALOMA-3.

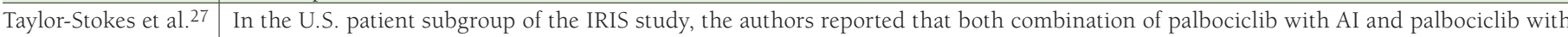
fulvestrant demonstrated favorable effectiveness. For the 360 patients treated with palbociclib and AI, the progression-free rate was 84.1\% and $64.3 \%$ for 12 and 24 months, respectively. For the 292 patients treated with palbociclib and fulvestrant, the 12-month progression-free rate was $79.8 \%$ overall, and the 12 -month survival rate was $87.9 \%$ overall, with no progression-free and survival rates available beyond 12 months.

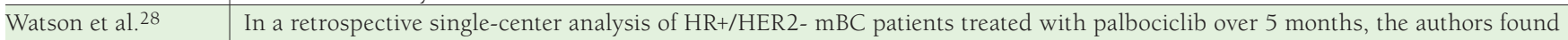
that $44 \%$ of the 64 patients had treatment deferrals due to neutropenia with a median time to first deferral of 4 weeks, while $23 \%$ requiring dose adjustments had no significant association with increased risk of disease progression. AEs were as expected, and only 3 patients discontinued treatment due to AEs, but 7 venous thromboembolic events were reported, which was higher than in PALOMA-3. Overall, compliance was good with existing CBC monitoring guidelines, with disease progression (n=30) as the main cause for discounting treatment.

\begin{tabular}{l|l}
\hline Kish et al.29 & The authors assessed the setting in which palbociclib was being initiated and examined the real-world occurrence of neutropenia
\end{tabular} and how providers monitored and managed these events. The study demonstrated a more heterogeneous line of therapy at initiation than RCTs, with 39.5\% of patients receiving palbociclib as first-line, $15.7 \%$ as second-line, $13.1 \%$ as third-line, and $31.7 \%$ as fourthline therapy or later. This study suggested good provider compliance with monitoring guidelines with a mean number of 6 CBC tests among all patients. Overall, $74.6 \%$ of patients had a neutropenic event, including $47.3 \%$ and $8.0 \%$ of patients with a grade 3 or 4 occurrence, respectively, data consistent with results in the phase 3 trials of PALOMA-2 and PALOMA-3.

\begin{tabular}{|c|c|}
\hline Stearns et al.30 & $\begin{array}{l}\text { The authors showed in a real-world EAP setting and with a median treatment duration of } 77 \text { days that the combination of palbociclib } \\
\text { and letrozole was generally well tolerated, and AEs were manageable by protocol-specified temporary treatment discontinuation, } \\
\text { dose reductions, and/or standard medical therapy. Interestingly, most patients reported "no problem" at baseline mobility, self-care, } \\
\text { and usual activities; however, nearly } 50 \% \text { reported "some problem" with respect to pain/discomfort and anxiety/depression. AEs } \\
\text { associated with permanent discontinuation of either or both study treatments were reported for } 10.2 \% \text { of the patients, and permanent } \\
\text { discontinuation of either or both study treatments were most commonly associated with disease progression ( } 22.2 \%) \text { and AEs ( } 8.1 \%) \text {. }\end{array}$ \\
\hline Xi et al. ${ }^{31}$ & $\begin{array}{l}\text { In a retrospective chart review of } 192 \text { postmenopausal patients with HR+/HER2- mBC, from electronic medical records from a } \\
\text { single-center institution, the authors analyzed treatment patterns and the effectiveness of palbociclib and subsequent regimens in } \\
\text { mBC. They found the median PFS on palbociclib was } 20.7 \text { months (first-line setting), } 12.8 \text { months (second-line), and } 4.0 \text { months in } \\
\text { subsequent-line settings, which was comparable to the PALOMA-2 and PALOMO-3 trials. }\end{array}$ \\
\hline Gong et al. ${ }^{32}$ & $\begin{array}{l}\text { The authors conducted a single institution, retrospective study of palbociclib and ET-related AEs. Out of the } 100 \text { patients included, } \\
\text { treatment resulted in dose modification in } 38.0 \% \text { of the patients due to AEs, with } 18.4 \% \text { requiring subsequent dose changes. Most } \\
\text { modifications occurred during the first } 2 \text { cycles, and } 54.8 \% \text { of them were due to grade } 3-4 \text { neutropenia. Notably, older age ( } \geq 65 \\
\text { years) did not affect palbociclib tolerance. }\end{array}$ \\
\hline Kuranz $^{33}$ & $\begin{array}{l}\text { The authors investigated mBC patients with HR+/HER2- breast cancer from a health care research network to compare the occurrence } \\
\text { of AEs in patients receiving palbociclib and letrozole with patients receiving AIs and in patients receiving palbociclib and fulvestrant } \\
\text { with patients on second-line AIs. Compared with ET, palbociclib demonstrated an increased risk of infection and bleeding. }\end{array}$ \\
\hline
\end{tabular}

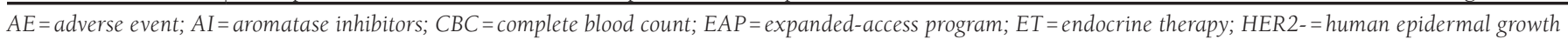
factor receptor 2-negative; $\mathrm{HR}+=$ hormone receptor-positive; $m B C=$ metastatic breast cancer; $P F S=$ progression-free survival; $R C T=$ randomized controlled trial.

decisions to initiate or continue cancer medications and can affect their adherence; those beliefs are multilayered with some of the most important influencing factors being the risk/benefit ratio, symptoms and side effects, and patient-provider relationships, ${ }^{18}$ as well as psychosocial factors (e.g., social support and knowledge of medication). ${ }^{7}$

A systematic review by Lin et al. (2017) identified psychosocial factors and barriers that may affect the adherence rate of breast cancer patients taking OAMs. ${ }^{7}$ Patient-provider relationships and positive views and beliefs of medication were the most widely reported effectors. Other important motivators identified by Lin et al. included depression and emotions, perception of illness, concern of side effects, self-efficacy in medication management and decision making, knowledge of medication, and social support on OAM adherence. Contrary to demographics or clinically related factors, these interpersonal 
and psychosocial factors constitute potentially modifiable effectors if the correct interventions are implemented to improve patient outcomes.

Brier et al. (2017) evaluated the relationship between health beliefs and adherence to AI in 437 postmenopausal women with HR+ breast cancer, using the Health Beliefs and Medication Adherence in Breast Cancer (HBMABC) scale and questionnaires. ${ }^{19}$ Of the participants, an estimated $21.3 \%$ were not adherent to therapy. Participants who perceived greater barriers to their AI treatments were less adherent (adjusted odds ratio $[\mathrm{aOR}]=1.71,95 \%$ confidence interval $[\mathrm{CI}]=1.03$ 2.86). This study highlights an important connection between adherence and how it may be influenced by perceived barriers using questionnaires to ascertain patient-reported outcomes.

Harbeck et al. (2016) assessed patient-reported outcomes (PROs) from the PALOMA-3 trial. ${ }^{20}$ On treatment with palbociclib plus fulvestrant (compared with fulvestrant plus placebo), overall quality-of-life (QoL) scores, assessed by the European Organisation for Research and Treatment of Cancer Qualityof-Life questionnaire (EORTC QLQ-C30), significantly favored the palbociclib plus fulvestrant group $(P=0.0313)$. This patient group also had significantly greater improvement from baseline in pain, while also significantly delayed deterioration in global QoL and pain compared with fulvestrant alone.

\section{Palbociclib Real-World Effectiveness and Safety}

Palbociclib in combination with an AI or fulvestrant was the first-in-class treatment and approved as a first-line agent in this setting of HR+/HER2- mBC. ${ }^{10}$ Palbociclib has demonstrated efficacy in clinical trials, ${ }^{21-24}$ but adherence issues and longterm efficacy and safety concerns are not always well captured in clinical trial settings. Several studies have attempted to demonstrate the reproducibility of evidence and external generalizability of randomized trials by assessing the real-world effectiveness and tolerability of palbociclib for advanced HR+/ HER2- patients with mBC (Table 1). ${ }^{25-33}$

Varella et al. (2019) conducted a real-world study looking at patients with an advanced HR+ breast cancer treated with palbociclib at the Cleveland Clinical health system from February 2015 to December $2017 .{ }^{25}$ In the 411 women included, the estimated overall median progression-free survival (PFS) for palbociclib/letrozole was 8.9 months. For palbociclib/fulvestrant, the estimated overall PFS was 10.3 months. The most common adverse events (AEs) were hematologic, with $8 \%$ of the patients permanently discontinuing palbociclib because of AEs. Bui et al. (2019) also aimed to assess the real-world effectiveness and tolerability of palbociclib combined with endocrine therapy in HR+/HER2- patients with mBC that progressed on previous endocrine therapy and to compare their results with the outcomes of the PALOMA-3 trial..$^{26}$ The median PFS in real-world clinical practice was 10.0 months compared with 9.5 months in PALOMA-3. Most AEs were managed with palbociclib dose.
Neutropenia was the most frequent grade 3-4 AE. Overall, the effectiveness and tolerability of palbociclib were similar to the results from PALOMA-3. In the U.S. patient subgroup of the IRIS study, Taylor-Stokes et al. (2019) reported that both a combination of palbociclib with AI and palbociclib with fulvestrant demonstrated favorable effectiveness. ${ }^{27}$ In a retrospective single-center analysis of HR+/HER2- mBC patients treated with palbociclib over 5 months, Watson et al. (2019) found that 44\% of the 64 patients had treatment deferrals due to neutropenia with a median time to first deferral of 4 weeks, while 23\% requiring dose adjustments had no significant association with increased risk of disease progression. ${ }^{28}$

Kish et al. (2018) assessed the setting in which palbociclib was being initiated and examined the real-world occurrence of neutropenia and how providers monitored and managed these events. ${ }^{29}$ This study suggested good provider compliance with monitoring guidelines. Overall, $74.6 \%$ of patients had a neutropenic event. Stearns et al. (2018) showed in a real-world expanded-access program (EAP) setting and with a median treatment duration of 77 days that the combination of palbociclib and letrozole was generally well tolerated and AEs were manageable by protocol-specified temporary treatment discontinuation, dose reductions, and/or standard medical therapy. ${ }^{30}$

In a retrospective chart review of 192 postmenopausal patients with HR+/HER2- mBC, from electronic medical records from a single-center institution, Xi et al. (2019) analyzed treatment patterns and the effectiveness of palbociclib and subsequent regimens in $\mathrm{mBC} \cdot{ }^{31}$ They found the median PFS on palbociclib was 20.7 months (first-line setting), 12.8 months (second-line), and 4.0 months in subsequent-line settings, which was comparable to the PALOMA-2 and PALOMO-3 trials. Gong et al. (2018) conducted a single institution, retrospective study of palbociclib and ET-related AEs. ${ }^{32}$ Out of the 100 patients included, treatment resulted in dose modification in $38.0 \%$ of the patients due to AEs, with $18.4 \%$ requiring subsequent dose changes. Most modifications occurred during the first 2 cycles, and $54.8 \%$ of them were due to grade 3-4 neutropenia. Kuranz (2018) investigated mBC patients with HR+/HER2- breast cancer from a health care research network. Compared to ET, palbociclib demonstrated an increased risk of infection and bleeding. ${ }^{33}$

Although informative, these were single-center/health care institution studies with small sample sizes (n varied from 30 to 411) and short follow-up time. Consequently, the generalizability of the results of these studies may be significantly reduced.

\section{Palbociclib Real-World Cost-Effectiveness}

Painful price points for payers seem to be more the rule than the exception regarding the newer add-ons for first-line therapies for HR+/HER2- breast cancer. These price points become more painful when there seem to be only marginal improvements in effectiveness over standards of care. Much of the evidence 
for the cost-effectiveness of palbociclib has been conducted outside of the United States. ${ }^{34-37}$ Furthermore, no study has considered the effect of adherence on cost-effectiveness using real-world data. Mamiya et al. (2017) found that, based on the U.S. societal perspective in both previously treated patients for metastatic breast cancer and treatment naive patients, palbociclib was highly unlikely ( $0 \%$ probability with a willingness to pay of $\$ 100,000$ per quality-adjusted life-year [QALY]) to be cost-effective compared with usual care. ${ }^{38}$ The authors used a discrete event simulation model using parameters based on published clinical trial data and other peer-reviewed studies.

A study by Mistry et al. (2018) evaluated letrozole to letrozole with palbociclib and letrozole with ribociclib, measured in life-years and QALYs, from a private third-party U.S. payer perspective. ${ }^{39}$ The authors used a partitioned survival model, which simulated lifetime costs and outcomes over a 40-year lifetime horizon. The main finding was that ribociclib with letrozole was dominant versus palbociclib with letrozole, with a cost savings of $\$ 43,037$ and gain of 0.086 QALYs. Bhattacharya et al. (2016) used a Markov disease-state transition model to compare the cost-effectiveness of the combination of letrozole with palbociclib, with mainstay therapies anastrozole and letrozole, in postmenopausal women with HR+/HER2- advanced breast cancer from a third-party payer perspective. ${ }^{40}$ Importantly, the transition state probabilities data were obtained from clinical trials and not real-world evidence, and no mention of adherence was made (only the abstract was available). The combination of palbociclib and letrozole was the least cost-effective with a cost-effectiveness value (in 2014 U.S. dollars) of $\$ 111,791.95$ compared with $\$ 14,226.94$ for anastrozole and $\$ 14,545.26$ for letrozole. Compared with anastrozole, the incremental costeffectiveness ratio of the combination therapy was $\$ 510,356.50$, while it was $\$ 21,824.34$ for letrozole. This could be indicative of the higher acquisition cost of palbociclib.

The studies described here show that palbociclib, at its current negotiated prices in foreign universal health care systems, as well as domestically in the United States, is not cost-effective compared with other available therapies. However, these existing studies do not incorporate real-world adherence data, which may hinder an accurate estimation of the value of oral anticancer medications, including palbociclib.

\section{Literature Gap and Future Directions}

To date, the study of the real-world effectiveness of palbociclib in the treatment of women with HR+/HER2- breast cancer has been limited. What is known hinges on randomized controlled trials (RCTs) data, which does not necessarily reflect realworld use and outcomes. To address this issue, researchers have attempted to bridge the divide between RCTs and realworld evidence. One such attempt by the American Society of Clinical Oncology (ASCO) focused on the development of a framework that incorporates clinical benefit and toxicity scores, with possible bonus points (as part of the advanced disease framework) to determine the net health benefit (NHB) score. ${ }^{41}$ The NHB score and associated costs of treatment are used to infer value gained for its degree of benefit. ASCO sought and received feedback on its conceptual value framework, leading to an update in May 2016. ${ }^{42}$ Within the update, a decision was made to adjust the clinical benefit component in the framework with a $20 \%$ reduction in points for RCTs that use surrogate endpoints, compared with RCTs, which use overall survival (OS). A study by Lakdawalla et al. (2017) assessed the difference between RCT efficacy and real-world effectiveness of cancer therapies and whether the type of endpoint used matters (i.e., surrogate vs. OS). ${ }^{43}$ The authors found that realworld and RCTs treatment benefits based on OS were similar; however, treatment benefits based on surrogate endpoints were $16 \%$ lower in the real world. Frameworks such as that of ASCO can help prescribers make more informed decisions with their patients by taking evidence from RCTs and translating it into a patient-specific strategy to fight breast cancer in the real-world setting. The ASCO framework needs to be applied in a variety of populations with cancer and tested for validity in defined populations, such as the $\mathrm{mBC}$ population.

Adherence is typically assumed to be $100 \%$ in RCTs, unless otherwise stated, because of strict control over the treatment process. This level of adherence is often not replicated in the real-world setting and thus creates the opportunity to explore this matter further. Racial and geographical differences among patients with $\mathrm{mBC}$ may also vary regarding adherence and access to care, but clinical trials often fail to enroll enough minority patients for a complete understanding to occur. This creates another opportunity to delve into this complex subject matter further. Adherence to therapy is also important from the payer perspective (e.g., Centers for Medicare \& Medicaid Services), since it is of interest for payers to know whether they, too, are dispensing funds for a therapy that will obtain desired outcomes in the real-world setting. With a more robust understanding of the real-world effectiveness of palbociclib, payers may more appropriately bargain prices of newer OAMs and, in effect, reduce the rise of oncologic drug costs while not hindering access for patients in need.

According to the willingness-to-pay thresholds from the United States, as previously discussed, the newer OAMs with AIs are not cost-effective relative to AIs alone ${ }^{38-40}$ As previously mentioned, these studies were based on RCTs, which may not be reflective of real-world conditions. Therefore, it is imperative that well-designed, comprehensive studies focusing on realworld cost-effectiveness (which includes adherence to therapy), racial and geographic disparities, and patient outcomes be undertaken to fill in the knowledge gaps for women with HR+/ HER2- mBC. This type of call to action has been championed by the National Institutes of Health, as stated in its Funding Opportunity Announcement Number PA-17-061.44 


\section{Real-World Clinical and Economic Outcomes Associated with Palbociclib for HR-Positive/HER2 Negative Metastatic Breast Cancer: A Commentary}

\section{Authors}

ARAM BABCOCK, PharmD, MBA, and VAKARAMOKO DIABY, PhD, Department of Pharmaceutical Outcomes and Policy, College of Pharmacy, University of Florida, Gainesville. ASKAL A. ALI, $\mathrm{PhD}$, Economic, Social \& Administrative Pharmacy, College of Pharmacy and Pharmaceutical Sciences, Florida AEM University, Tallahassee. RAJESH BALKRISHNAN, PhD, School of Medicine and School of Nursing, University of Virginia, Charlottesville. ALBERTO J. MONTERO, MD, MBA, Department of Hematology/Oncology, Taussig Cancer Institute, Cleveland Clinic, Cleveland, Ohio.

AUTHOR CORRESPONDENCE: Vakaramoko Diaby, PhD, University of Florida College of Pharmacy, 1225 Centre Dr., Rm. 3317, Gainesville, FL 32610. Tel.: 352.273.9397;

E-mail:v.diaby@cop.ufl.edu.

\section{DISCLOSURES}

No funding supported the writing of this article. The authors have nothing to disclose.

\section{REFERENCES}

1. Amercian Cancer Society. How common is breast cancer? 2020. Available at: https://www.cancer.org/cancer/breast-cancer/about/how-common-isbreast-cancer.html. Accessed April 14, 2020

2. Breastcancer.org. Molecular subtypes of breast cancer. 2019. Available at: https://www.breastcancer.org/symptoms/types/molecular-subtypes. Accessed April 14, 2020

3. Sullivan R, Peppercorn J, Sikora K, et al. Delivering affordable cancer care in high-income countries. Lancet Oncol. 2011;12(10):933-80.

4. Rashidian A, Barfar E, Hosseini H, Nosratnejad S, Barooti E. Cost effectiveness of breast cancer screening using mammography; a systematic review. Iran J Public Health. 2013;42(4):347-57.

5. Zelle SG, Baltussen RM. Economic analyses of breast cancer control in low- and middle-income countries: a systematic review. Syst Rev. 2013;2(1):20

6. Eggersmann TK, Degenhardt T, Gluz O, Wuerstlein R, Harbeck N. CDK4/6 inhibitors expand the therapeutic options in breast cancer: palbociclib, ribociclib and abemaciclib. BioDrugs. 2019;33(2):125-35.

7. Lin C, Clark R, Tu P, Bosworth HB, Zullig LL. Breast cancer oral anticancer medication adherence: a systematic review of psychosocial motivators and barriers. Breast Cancer Res Treat. 2017;165(2):247-60.

8. Light DW, Kantarjian H. Market spiral pricing of cancer drugs. Cancer. 2013;119(22):3900-02

9. Vivot A, Jacot J, Zeitoun J-D, Ravaud P, Crequit P, Porcher R. Clinical benefit, price and approval characteristics of FDA-approved new drugs for treating advanced solid cancer, 2000-2015. Ann Oncol. 2017;28(5):1111-16.

10. U.S. Food and Drug Administration. Palbociclib (IBRANCE). March 31, 2017. Available at: https://www.fda.gov/drugs/resources-informationapproved-drugs/palbociclib-ibrance. Accessed April 14, 2020.

11. Greer JA, Amoyal N, Nisotel L, et al. A systematic review of adherence to oral antineoplastic therapies. Oncologist. 2016;21(3):354-76.

12. Given BA, Spoelstra SL, Grant M. The challenges of oral agents as antineoplastic treatments. Semin Oncol Nurs. 2011;27(2):93-103.
13. Ganesan P, Sagar TG, Dubashi B, et al. Nonadherence to imatinib adversely affects event free survival in chronic phase chronic myeloid leukemia. Am J Hematol. 2011;86(6):471-74.

14. Hershman DL, Shao T, Kushi LH, et al. Early discontinuation and nonadherence to adjuvant hormonal therapy are associated with increased mortality in women with breast cancer. Breast Cancer Res Treat. 2011;126(2):529-37.

15. Makubate B, Donnan PT, Dewar JA, Thompson AM, McCowan C. Cohort study of adherence to adjuvant endocrine therapy, breast cancer recurrence and mortality. Br J Cancer. 2013;108(7):1515-24.

16. McCowan C, Wang S, Thompson AM, Makubate B, Petrie DJ. The value of high adherence to tamoxifen in women with breast cancer: a communitybased cohort study. Br J Cancer. 2013;109(5):1172-80.

17. Wu EQ, Johnson S, Beaulieu N, et al. Healthcare resource utilization and costs associated with non-adherence to imatinib treatment in chronic myeloid leukemia patients. Curr Med Res Opin. 2010;26(1):61-69.

18. Marshall V, Given B. Factors associated with medication beliefs in patients with cancer: an integrative review. Oncol Nurs Forum. 2018;45(4):508-26.

19. Brier MJ, Chambless DL, Gross R, Chen J, Mao JJ. Perceived barriers to treatment predict adherence to aromatase inhibitors among breast cancer survivors. Cancer. 2017;123(1):169-76.

20. Harbeck N, Iyer S, Turner N, et al. Quality of life with palbociclib plus fulvestrant in previously treated hormone receptor-positive, HER2-negative metastatic breast cancer: patient-reported outcomes from the PALOMA-3 trial. Ann Oncol Off J Eur Soc Med Oncol. 2016;27(6):1047-54.

21. Finn RS, Martin M, Rugo HS, et al. Palbociclib and letrozole in advanced breast cancer. N Engl J Med. 2016;375(20):1925-36.

22. Rugo HS, Finn RS, Diéras V, et al. Palbociclib plus letrozole as first-line therapy in estrogen receptor-positive/human epidermal growth factor receptor 2-negative advanced breast cancer with extended follow-up. Breast Cancer Res Treat. 2019;174(3):719-29.

23. Turner NC, Ro J, André F, et al. Palbociclib in hormone-receptor-positive advanced breast cancer. N Engl J Med. 2015;373(3):209-19.

24. Cristofanilli M, Turner NC, Bondarenko I, et al. Fulvestrant plus palbociclib versus fulvestrant plus placebo for treatment of hormone-receptor-positive, HER2-negative metastatic breast cancer that progressed on previous endocrine therapy (PALOMA-3): final analysis of the multicentre, doubleblind, phas. Lancet Oncol. 2016;17(4):425-39.

25. Varella L, Eziokwu AS, Jia X, et al. Real-world clinical outcomes and toxicity in metastatic breast cancer patients treated with palbociclib and endocrine therapy. Breast Cancer Res Treat. 2019;176(2):429-34.

26. Bui TBV, Burgers DM, Agterof MJ, van de Garde EM. Real-world effectiveness of palbociclib versus clinical trial results in patients with advanced/ metastatic breast cancer that progressed on previous endocrine therapy. Breast Cancer (Auckl). 2019;13:117822341882323. Available at: https://www. ncbi.nlm.nih.gov/pmc/articles/PMC6330732/. Accessed April 14, 2020.

27. Taylor-Stokes G, Mitra D, Waller J, Gibson K, Milligan G, Iyer S Treatment patterns and clinical outcomes among patients receiving palbociclib in combination with an aromatase inhibitor or fulvestrant for HR+/ HER2-negative advanced/metastatic breast cancer in real-world settings in the US: Results from the IRIS study. Breast. 2019;43:22-27.

28. Watson GA, Deac O, Aslam R, et al. Real-world experience of palbociclib-induced adverse events and compliance with complete blood count monitoring in women with hormone receptor-positive/HER2-negative metastatic breast cancer. Clin Breast Cancer. 2019;19(1):e186-e194.

29. Kish JK, Ward MA, Garofalo D, et al. Real-world evidence analysis of palbociclib prescribing patterns for patients with advanced/metastatic breast cancer treated in community oncology practice in the USA one year post approval. Breast Cancer Res. 2018;20(1):37. 


\section{Real-World Clinical and Economic Outcomes Associated with Palbociclib for HR-Positive/HER2 Negative Metastatic Breast Cancer: A Commentary}

30. Stearns V, Brufsky AM, Verma S, et al. Expanded-access study of palbociclib in combination with letrozole for treatment of postmenopausal women with hormone receptor-positive, HER2-negative advanced breast cancer. Clin Breast Cancer. 2018;18(6):e1239-e1245.

31. Xi J, Oza A, Thomas S, et al. Retrospective analysis of treatment patterns and effectiveness of palbociclib and subsequent regimens in metastatic breast cancer. J Natl Compr Cancer Netw. 2019;17(2):141-47.

32. Gong J, Cho M, Yu KW, Waisman J, Yuan Y, Mortimer J. A single institution experience with palbociclib toxicity requiring dose modifications. Breast Cancer Res Treat. 2018;168(2):381-87.

33. Kuranz S. PCN3 - Real world evidence of metastatic breast cancer treatment: a comparison of adverse effects between palbociclib treatment and endocrine therapy [abstract]. Value Health. 2018;21(Suppl 3):S15. Available at: https://www.valueinhealthjournal.com/article/S1098-3015(18)33386-2/ fulltext. Accessed April 14, 2020.

34. Matter-Walstra K, Ruhstaller T, Klingbiel D, Schwenkglenks M, Dedes KJ. Palbociclib as a first-line treatment in oestrogen receptor-positive, HER2negative, advanced breast cancer not cost-effective with current pricing: a health economic analysis of the Swiss Group for Clinical Cancer Research (SAKK). Breast Cancer Res Treat. 2016;158(1):51-57.

35. Matter-Walstra K, Schwenkglenks M, Dedes KJ. Cost-effectiveness of palbociclib plus letrozole versus letrozole alone as a first-line treatment in women with oestrogen receptor-positive, HER2-negative, advanced breast cancer. Revised results for the Swiss health care setting. Breast Cancer Res Treat. 2017;163(3):635-35.

36. Raphael J, Helou J, Pritchard KI, Naimark DM. Palbociclib in hormone receptor positive advanced breast cancer: a cost-utility analysis. Eur J Cancer. 2017;85:146-54.

37. Galve-Calvo E, González-Haba E, Gostkorzewicz J, Martínez I, PérezMitru A. Cost-effectiveness analysis of ribociclib versus palbociclib in the first-line treatment of HR+/HER2- advanced or metastatic breast cancer in Spain. Clinicoecon Outcomes Res. 2018;10:773-90
38. Mamiya H, Tahara RK, Tolaney SM, Choudhry NK, Najafzadeh M. Costeffectiveness of palbociclib in hormone receptor-positive advanced breast cancer. Ann Oncol. 2017;28(8):1825-31.

39. Mistry R, May JR, Suri G, et al. Cost-effectiveness of ribociclib plus letrozole versus palbociclib plus letrozole and letrozole monotherapy in the first-line treatment of postmenopausal women with HR+/HER2- advanced or metastatic breast cancer: a U.S. payer perspective. J Manag Care Spec Pharm. 2018;24(6):514-23. Available at: https://www.jmcp.org/doi/10.18553/ jmcp.2018.24.6.514

40. Bhattacharya K, Yang Y. A cost-effectiveness analysis of palbociclib and other aromatase inhibitors for treatment of advanced breast cancer [abstract]. Value Health. 2016;19(3):A150. Available at: https://www.valueinhealthjournal.com/article/S1098-3015(16)01657-0/abstract. Accessed April 14, 2020.

41. Schnipper LE, Davidson NE, Wollins DS, et al. American Society of Clinical Oncology statement: a conceptual framework to assess the value of cancer treatment options. J Clin Oncol. 2015;33(23):2563-77.

42. Schnipper LE, Davidson NE, Wollins DS, et al. Updating the American Society of Clinical Oncology value framework: revisions and reflections in response to comments received. J Clin Oncol. 2016;34(24):2925-34.

43. Lakdawalla DN, Shafrin J, Hou N, et al. Predicting real-world effectiveness of cancer therapies using overall survival and progression-free survival from clinical trials: empirical evidence for the ASCO value framework. Value Health. 2017;20(7):866-75

44. National Institutes of Health. PA-17-061: Oral anticancer agents: utilization, adherence, and health care delivery. Exploratory/developmental research grant (R21). Available at: https://grants.nih.gov/grants/guide/pafiles/PA-17-061.html. Accessed April 14, 2020. 